9-2003

\title{
Geometry and Topology of Escape. I. Epistrophes
}

\author{
K. A. Mitchell \\ William \& Mary \\ J. P. Handley \\ William \& Mary \\ B. Tighe \\ William \& Mary \\ John B. Delos \\ William \& Mary, jbdelos@wm.edu \\ Stephen Knudson
}

Follow this and additional works at: https://scholarworks.wm.edu/aspubs

Part of the Physics Commons

\section{Recommended Citation}

Mitchell, K. A.; Handley, J. P.; Tighe, B.; Delos, John B.; and Knudson, Stephen, Geometry and Topology of Escape. I. Epistrophes (2003). Chaos: An Interdiscipliary Journal of Nonlinear Science, 13(3), 880-891. https://doi.org/10.1063/1.1598311

This Article is brought to you for free and open access by the Arts and Sciences at W\&M ScholarWorks. It has been accepted for inclusion in Arts \& Sciences Articles by an authorized administrator of W\&M ScholarWorks. For more information, please contact scholarworks@wm.edu. 


\title{
Geometry and topology of escape. I. Epistrophes
}

\author{
K. A. Mitchell, ${ }^{\text {a) }}$ J. P. Handley, B. Tighe, and J. B. Delos ${ }^{\text {b) }}$ \\ Department of Physics, College of William and Mary, Williamsburg, Virginia 23187-8795 \\ S. K. Knudson \\ Department of Chemistry, College of William and Mary, Williamsburg, Virginia 23187-8795
}

(Received 1 October 2002; accepted 17 June 2003; published 15 August 2003)

\begin{abstract}
We consider a dynamical system given by an area-preserving map on a two-dimensional phase plane and consider a one-dimensional line of initial conditions within this plane. We record the number of iterates it takes a trajectory to escape from a bounded region of the plane as a function along the line of initial conditions, forming an "escape-time plot." For a chaotic system, this plot is in general not a smooth function, but rather has many singularities at which the escape time is infinite; these singularities form a complicated fractal set. In this article we prove the existence of regular repeated sequences, called "epistrophes," which occur at all levels of resolution within the escape-time plot. (The word "epistrophe" comes from rhetoric and means "a repeated ending following a variable beginning.") The epistrophes give the escape-time plot a certain self-similarity, called "epistrophic" self-similarity, which need not imply either strict or asymptotic self-similarity. (C) 2003 American Institute of Physics. [DOI: 10.1063/1.1598311]
\end{abstract}

Chaotic transport, and the escape of trajectories from defined regions of phase space, has been an important topic in dynamics for many years, because it describes phenomena that occur in many branches of physics. For example, some meteorites that fell on Antarctica are believed to have come from Mars; how they escaped from Mars' gravitational field is a problem in the theory of chaotic transport. ${ }^{1}$ At a smaller scale, one of the important topics in nanophysics is ballistic transport of electrons through a small junction: electrons enter a junction from one lead, bounce around within the junction following either regular or chaotic paths, and eventually find their way to an exit lead. ${ }^{2}$ A closely related problem is chaotic propagation of light rays in a distorted cylindrical glass bead. ${ }^{3}$ At the molecular level, we may think about the breakup of a temporarily bound complex, such as a He atom weakly bound to an $I_{2}$ molecule. ${ }^{4-7}$ At the atomic level, the ionization of an excited hydrogen atom in applied electric and magnetic fields is an ideal candidate for the laboratory study of chaotic transport. ${ }^{8-14} \mathrm{We}$ can learn many of the properties of chaotic transport by studying area-preserving maps of the plane. We examine the time required to escape from a specified region of the plane, plotted as a function along a given line of initial conditions. Within this escape-time plot, we study regular sequences of escaping intervals, which we call "epistrophes."

\section{INTRODUCTION}

We are motivated by the chaotic ionization of a hydrogen atom placed in strong parallel electric and magnetic fields. The dynamics of the hydrogenic electron can be mod-

${ }^{a)}$ Electronic mail: kevinm@physics.wm.edu

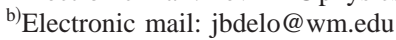

eled classically by an area-preserving map on a twodimensional phase plane. This map exhibits a prominent homoclinic tangle (see the following), which organizes the dynamics, leading to phase space transport and eventually escape. The mechanism of escape via a tangle is a common model for many classical systems. In this paper, we consider the general problem of escape for an arbitrary map possessing a homoclinic tangle exhibiting the basic structure shown in Fig. 1.

The map in Fig. 1 has an unstable fixed point ( $X$ point) $\mathbf{z}_{X}$, with a pair of stable and unstable manifolds attached to it. These manifolds are invariant curves containing all points that asymptote to $\mathbf{z}_{\mathbf{X}}$ under forward and backward iterates, respectively. ${ }^{15,16}$ The curves intersect transversely at the point $\mathbf{P}_{0}$. The "complex" is the region bounded by the segments of the stable and unstable manifolds joining $\mathbf{z}_{X}$ to $\mathbf{P}_{0}$; escape is defined as mapping out of the complex. As explained by Poincaré, the transverse intersection $\mathbf{P}_{0}$ produces a homoclinic tangle. Between successive intersection points $\mathbf{P}_{n}$ and $\mathbf{Q}_{n}$, the manifolds bound lobes denoted $E_{n}$ and $C_{n}$ in Fig. 1. As these lobes are mapped forward or backward, their widths are compressed and their lengths are stretched; they become long and thin and develop intricate twisted shapes. The resulting complex structure of the intersecting stable and unstable manifolds is called a homoclinic tangle.

An important aspect of any classical decay problem is the distribution of initial points in phase space. When modeling the breakup of molecular collision complexes, for example, one normally assumes that the complex is more-orless in thermal equilibrium. A microcanonical distribution of initial probability might be used within the collision complex, with equal probabilities in equal areas, or perhaps some other smooth distribution. However, in most experiments on excited atoms in strong fields, the initial distribution is quite different. The electron attains a high energy by single-photon excitation from a localized strongly bound initial state. ${ }^{17-22}$ 


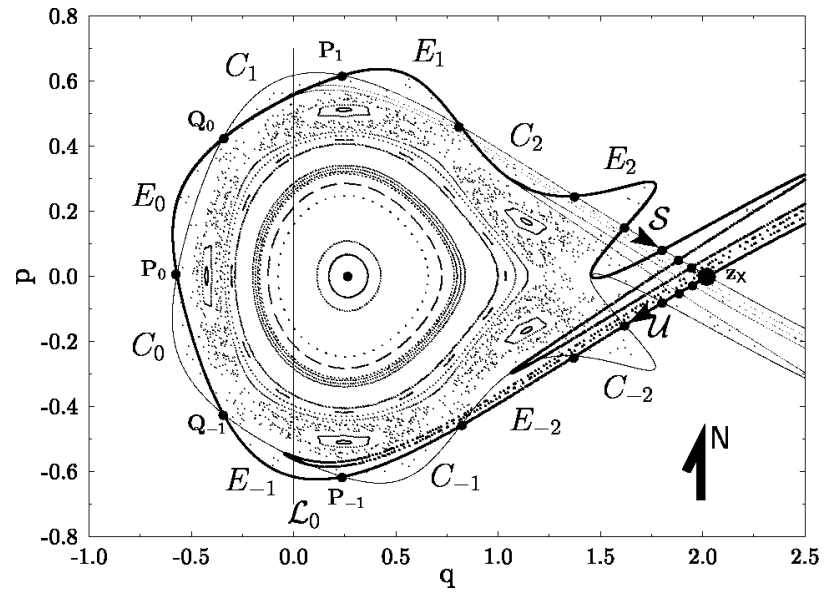

FIG. 1. A phase space portrait is shown for a map possessing a single homoclinic tangle. [The map is defined by Eqs. (A1)-(A3) with $\tau=1.5, f$ $=0.25$, and $m=0.57$.] The $\mathbf{X}$ point $\mathbf{z}_{\mathbf{x}}$ has a stable manifold $\mathcal{S}$ and unstable manifold $\mathcal{U}$ which cross repeatedly to form the tangle. The primary intersection point $\mathbf{P}_{0}$ defines the complex, the northern and southern boundaries of which are the segments of $\mathcal{S}$ and $\mathcal{U}$ joining $\mathbf{P}_{0}$ to $\mathbf{z}_{\mathrm{X}}$. Orbits escape the complex by mapping from $E_{-1}$ into $E_{0}$ and then move away through successive iterates: $E_{1}, E_{2}, E_{3}, \ldots$. Orbits are captured by mapping from $C_{0}$ into $C_{1}$. The line of initial conditions $\mathcal{L}_{0}$ coincides with $q=0$.

The trajectories therefore start close to the nucleus and go out in all directions. This initial distribution is well modeled by assuming that all electrons begin exactly at the nucleus, with constant energy, and with a smooth distribution of outgoing directions. ${ }^{23-25}$ In the phase plane, this yields a distribution of initial states along a line of initial conditions. Thus, we assume that the initial distribution of states in phase space lies along some curve $\mathcal{L}_{0}$, the details of which depend on the problem at hand. (See Fig. 1.) Along this curve there is some initial density of points; for hydrogen this density represents the initial angular distribution of outgoing electrons. $^{26,56}$

We plot the number of iterates $n_{i}$ needed to escape as a function along the line of initial conditions $\mathcal{L}_{0}$, forming an escape-time plot, as shown in Fig. 2. Each line segment in Fig. 2 represents an interval, or escape segment, of $\mathcal{L}_{0}$, in which all points escape the complex at the same iterate. The escape-time plot is clearly a very complicated function with "fractal" properties; this fractal structure is created by the repeated intersections of the stable manifold with the line of initial conditions. Our objective is to describe certain regular structures within this plot.

Figure 2 contains many prominent sequences of escape segments, several of which are indicated by bold arrows. We call each such sequence an epistrophe. Epistrophes have several important properties. (1) Beginning at some initial iterate, each epistrophe contains one escape segment at every subsequent iterate. (2) Each epistrophe converges to some point on $\mathcal{L}_{0}$. (3) Within a given epistrophe, the lengths of the escape segments decrease geometrically (in the limit $n_{i}$ $\rightarrow \infty$ ) with the ratio of successive lengths converging to the Liapunov factor (i.e., the largest eigenvalue) $\alpha$ of the $\mathrm{X}$ point. This is true regardless of which epistrophe we analyze.

The epistrophes form hierarchical sequences-we see in Fig. 2 that the end points of each escape segment serve as the

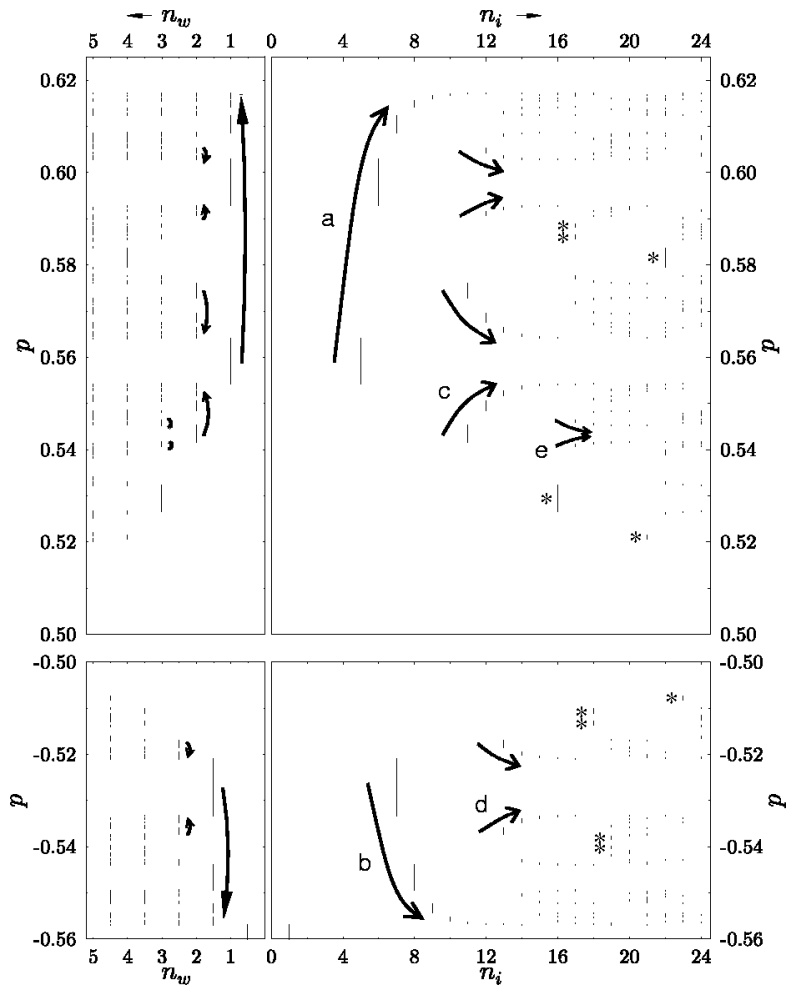

FIG. 2. Escape data $n_{i}$ and $n_{w}$ are plotted for the map in Fig. 1. Shown on the right is the number of iterates $n_{i}$ required for a point to escape from the complex; it is plotted as a function of $p$ parametrizing the line of initial conditions $\mathcal{L}_{0}$. Several sequences (epistrophes) of escape segments are indicated with bold arrows. Several escape segments (strophes) are marked by asterisks; these segments are not easily predicted from the current level of theory. Plotted on the left is the winding number of the trajectory as it escapes to infinity.

limit points for epistrophes beginning at higher iterate number. For example, consider epistrophe a, which begins at $n_{i}$ $=5$ (around $p=0.56$ ) and progresses upward, containing segments at $n_{i}=6,7,8, \ldots$. Upon each of the two end points of the first segment $\left(n_{i}=5\right)$, there converges another epistrophe which begins at $n_{i}=11$. Similarly, the second segment of epistrophe a $\left(n_{i}=6\right)$ has an epistrophe converging upon each of its end points, beginning at $n_{i}=12$. In fact, every escape segment has an epistrophe which converges upon each of its end points. Thus, epistrophes appear throughout the escape-time plot and on all scales.

The main result of this paper is the Epistrophe Theorem (Sec. III), which proves and elaborates upon the above-noted observations for an arbitrary homoclinic tangle and an arbitrary line of initial conditions. The beginning of each epistrophe is not described by the Epistrophe Theorem, leaving a certain unpredictability in how an epistrophe starts. What is described is the asymptotic behavior of the tail of the epistrophe. In fact, we prove that, up to an overall rescaling, the asymptotic tails of all epistrophes are identical; we characterize these tails with geometric quantities $(\alpha, \chi$, and $\phi$ in Theorem 1).

The recursive nature of the Epistrophe Theorem and the scaling relation between the epistrophe tails implies a certain self-similarity to the escape-time plot. However, the Epistrophe Theorem is itself not strong enough to imply true self- 
similarity (or even asymptotic self-similarity). Our data indicate that there may be numerous escape segments, which we call "strophes," that do not belong to any epistrophe and that even tend to dominate the escape-time plot at long times. Several such strophes are indicated by asterisks in Fig. 2. We can thus say that the Epistrophe Theorem implies a kind of "epistrophic" self-similarity: epistrophes (self-similar sequences) occur on all scales, but there may also be additional segments, or strophes, that persist (and may even dominate) in the asymptotic limit.

On the left-hand side of Fig. 2 is plotted the winding number $n_{w}$ of escape, i.e., the number of times a trajectory winds around the "center" of the complex as it escapes to infinity. (In this case the center is the stable zone in Fig. 1.) The data show that the winding number $n_{w}$ is constant along an epistrophe. For example, all escape segments in epistrophe a have $n_{w}=1$; all segments in epistrophe $\mathbf{d}$ have $n_{w}$ $=2.5$, and so on. Also, the winding number of an epistrophe is always one greater than the winding number of the segment upon which it converges. For example, epistrophe $\mathbf{c}$ has $n_{w}=2$ and converges upon a segment of a with $n_{w}=1$. In a separate publication, we will prove several theorems explaining these observations.

The impact of tangles on chaotic transport has been an active field of research for at least the last 20 years, with notable contributions by MacKay, Meiss, and Percival, ${ }^{27}$ Davis and Gray, ${ }^{4}$ Rom-Kedar, ${ }^{28,29}$ Wiggins, ${ }^{15}$ and numerous others. More specifically, there has been significant interest in the fractal behavior of escape-time plots (or alternatively, scattering functions) in a variety of fields, including work by Noid et al. ${ }^{30}$ Petit and Henon, ${ }^{31}$ Eckhardt, ${ }^{32,33}$ Jung and co-workers, ${ }^{34-39}$ and Gaspard and co-workers. ${ }^{40,41}$

Our research was inspired by the work of Tiyapan and Jaffé on the scattering of $\mathrm{He}$ from an excited $\mathrm{I}_{2}$ dimer. ${ }^{5-7} \mathrm{In}$ their study (particularly Ref. 5), Tiyapan and Jaffé examined a final-action versus initial-angle plot (analogous to the escape-time plot) over a wide range of scales. In their numerical data, they identified infinite sequences equivalent to our epistrophes. Jung and co-workers have also extensively studied scattering functions. In particular, they partially label the asymptotically bound orbits using a symbolic dynamics, which captures important topological structures of the scattering functions. As in the work of Tiyapan and Jaffé, the sequences which we call epistrophes are present in their description. However, none of the above-mentioned work gives a full characterization of the epistrophes, nor does it give a proof that all epistrophes of a given map are asymptotically self-similar and asymptotically similar to each other. This is the primary result of the present paper and is summarized in the Epistrophe Theorem. The Epistrophe Theorem is also closely related to (but distinct from) Palis's " $\lambda$-lemma." 42,43

Our paper has the following structure. Section II states the technical assumptions we require of the tangle. Section III contains the Epistrophe Theorem. Implications for the fractal structure of the escape-time plots are discussed in Secs. III B-III D. Section IV contains conclusions and a discussion of future work. Appendix A defines our example map. The proof of the Epistrophe Theorem is contained in Appendix B.

\section{HOMOCLINIC TANGLES}

\section{A. Assumptions on the map}

We consider an arbitrary "saddle-center map" $\mathcal{M}$ which has a simple homoclinic tangle (as shown in Fig. 1) that is described by the following five assumptions.

Assumption 1: The map $\mathcal{M}$ is a canonical map or, more precisely, an analytic area- and orientation-preserving diffeomorphism of an open subset of the phase plane.

Assumption 2: The map has an unstable fixed point $(\mathrm{X}$ point) $\mathbf{z}_{\mathbf{X}}$, without inversion.

Assumption 3: Proceeding away from the $\mathrm{X}$ point, one branch of the stable manifold and one branch of the unstable manifold (called the nontangled manifolds) each go to infinity without intersecting any other stable or unstable manifold; the other branch $\mathcal{S}$ of the stable manifold and $\mathcal{U}$ of the unstable manifold (called the tangled manifolds) intersect each other transversely.

Assumption 3 is essentially Rom-Kedar's definition of an "open map." ${ }^{29}$ A primary intersection point, or "pip," $\mathbf{z}_{\text {pip }}$ is a transverse intersection between $\mathcal{S}$ and $\mathcal{U}$ such that the segment of $\mathcal{S}$ joining $\mathbf{z}_{\mathbf{X}}$ to $\mathbf{z}_{\text {pip }}$ does not intersect the segment of $\mathcal{U}$ joining $\mathbf{z}_{\mathrm{X}}$ to $\mathbf{z}_{\text {pip }}{ }^{15,44}$ We choose a pip $\mathbf{P}_{0}$ such that $\mathcal{U}$ crosses $\mathcal{S}$ from right to left (using the natural orientation of $\mathcal{U}$ and $\mathcal{S}$, as in Fig. 1). The complex is defined as the region enclosed by $\mathcal{S}$ and $\mathcal{U}$ from $\mathbf{z}_{\mathrm{X}}$ to $\mathbf{P}_{0}$; it contains its boundary, including $\mathbf{z}_{\mathbf{X}}$. The forward and backward iterates of $\mathbf{P}_{0}$ are also homoclinic intersections (with the same sense) and are denoted $\mathbf{P}_{n}=\mathcal{M}^{n}\left(\mathbf{P}_{0}\right),-\infty<n<\infty$.

Assumption 4: Between $\mathbf{P}_{0}$ and $\mathbf{P}_{1}, \mathcal{S}$ and $\mathcal{U}$ intersect just once, at a point we call $\mathbf{Q}_{0}$.

The intersection $\mathbf{Q}_{0}$ has the opposite sense as $\mathbf{P}_{0}$, as do its forward and backward iterates $\mathbf{Q}_{n}=\mathcal{M}^{n}\left(\mathbf{Q}_{0}\right),-\infty<n$ $<\infty$. The segments of $\mathcal{S}$ and $\mathcal{U}$ between $\mathbf{P}_{0}$ and $\mathbf{Q}_{0}$ enclose the escape zone $E_{0}$, which by definition contains its outer $\mathcal{U}$ boundary but not its inner $\mathcal{S}$ boundary (and neither $\mathbf{P}_{0}$ nor $\mathbf{Q}_{0}$ ). Similarly, the segments of $\mathcal{S}$ and $\mathcal{U}$ between $\mathbf{Q}_{-1}$ and $\mathbf{P}_{0}$ enclose the capture zone $C_{0}$, which by definition contains its outer $\mathcal{S}$ boundary but not its inner $\mathcal{U}$ boundary (and neither $\mathbf{P}_{0}$ nor $\left.\mathbf{Q}_{-1}\right)$. The forward and backward iterates of $E_{0}$ and $C_{0}$ are called the escape zones $E_{n}$ and the capture zones $C_{n}(-\infty<n<\infty)$.

The lobes $C_{0}$ and $E_{-1}$ form a turnstile: ${ }^{15,27}$ on one iterate of the map, all points in $C_{0}$ map into the complex, i.e., are captured, and all points in $E_{-1}$ map out of the complex, i.e., escape. All points that eventually escape the complex lie in some escape zone $E_{-k}=\mathcal{M}^{-k}\left(E_{0}\right), k>0$.

Assumption 5: Mapping forward causes all points in $E_{0}$ to march off to infinity, never to re-enter the complex. Likewise mapping backward causes all points in $C_{0}$ to march off to infinity, never to re-enter.

Assumption 5 means that no point can escape from and subsequently return to the complex; equivalently, $E_{n} \cap C_{n^{\prime}}$ $=\varnothing$ for $n \geqslant 0$ and $n^{\prime} \leqslant 0$.

By convention we orient the tangle in the $p q$ plane as shown in Figs. 1 and 3; that is, $\mathbf{P}_{0}$ is west of $\mathbf{z}_{X}$, and $\mathcal{S}$ is north of $\mathcal{U}$ when linearized about $\mathbf{z}_{\mathrm{X}}$. 


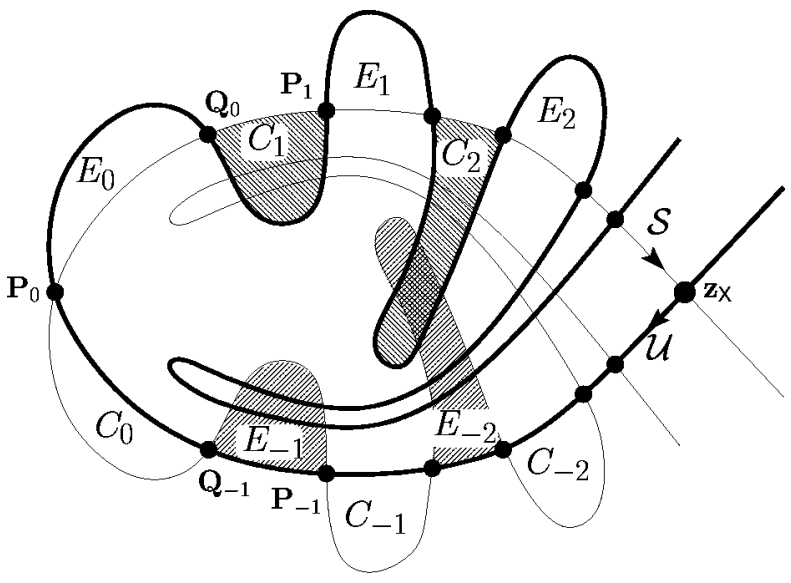

FIG. 3. A qualitative representation of a homoclinic tangle satisfying Assumptions 1-5. It displays features similar to the data in Fig. 1, but the escape zones and capture zones are more clearly labeled.

\section{B. Canonical length on the stable and unstable manifolds}

For any point $\mathbf{z}_{0} \in \mathcal{S}$, there is a natural length $s\left(\mathbf{z}_{0}\right)$ along the stable manifold, as measured from $\mathbf{z}_{\mathrm{X}}$. Setting $\mathbf{z}_{n}$ $=\mathcal{M}^{n}\left(\mathbf{z}_{0}\right)$, we define

$$
s\left(\mathbf{z}_{0}\right)=\lim _{n \rightarrow \infty}\left|\mathbf{z}_{n}-\mathbf{z}_{\mathbf{X}}\right| \alpha^{n},
$$

where $\|$ is the standard Euclidean vector norm. The limit in Eq. (1) is well-defined since after each iterate, $\left|\mathbf{z}_{n}-\mathbf{z}_{\mathbf{X}}\right|$ decreases by a factor $\alpha$ within the linear approximation to $\mathcal{M}$. It follows from Eq. (1) that

$$
s\left(\mathbf{z}_{0}\right)=s\left(\mathbf{z}_{n}\right) \alpha^{n} .
$$

Up to a constant scale factor, Eq. (2) uniquely determines $s$. Under a canonical transformation, the function $s(\mathbf{z})$ only changes by an overall scale factor. Hence, we call $s$ the $c a$ nonical length along $\mathcal{S}$, and we call $\left|s(\mathbf{z})-s\left(\mathbf{z}^{\prime}\right)\right|$ the canonical length between $\mathbf{z}$ and $\mathbf{z}^{\prime}$. $\mathcal{U}$ by

Analogous to $s$, we define the canonical length $u$ along

$$
u\left(\mathbf{z}_{0}\right)=\lim _{n \rightarrow \infty}\left|\mathbf{z}_{-n}-\mathbf{z}_{\mathbf{X}}\right| \alpha^{n},
$$

which satisfies

$$
u\left(\mathbf{z}_{0}\right)=u\left(\mathbf{z}_{-n}\right) \alpha^{n} .
$$

Recall that the lobes $E_{n}$ and $C_{n}$ each have a $\mathcal{U}$ boundary and an $\mathcal{S}$ boundary. We denote the canonical lengths of these boundaries by

$$
\begin{aligned}
& u\left(E_{0}\right)=\left|u\left(\mathbf{Q}_{0}\right)-u\left(\mathbf{P}_{0}\right)\right|, \\
& s\left(E_{0}\right)=\left|s\left(\mathbf{Q}_{0}\right)-s\left(\mathbf{P}_{0}\right)\right|, \\
& u\left(C_{0}\right)=\left|u\left(\mathbf{P}_{0}\right)-u\left(\mathbf{Q}_{-1}\right)\right|, \\
& s\left(C_{0}\right)=\left|s\left(\mathbf{P}_{0}\right)-s\left(\mathbf{Q}_{-1}\right)\right| .
\end{aligned}
$$

\section{The curve of initial conditions}

We choose a (differentiable) curve of initial conditions $\mathcal{L}_{0}$ that passes through the complex. We introduce on $\mathcal{L}_{0}$ the
Euclidean line element $d \lambda=\left(d p^{2}+d q^{2}\right)^{1 / 2}$, which gives us a coordinate $\lambda$ on $\mathcal{L}_{0}$ and a length $\lambda_{a b}=\left|\lambda_{a}-\lambda_{b}\right|$ of the segment between end points $\lambda=\lambda_{a}$ and $\lambda=\lambda_{b}$. We allow for an arbitrary positive density $d \mu=\rho(\lambda) d \lambda$ of initial points on $\mathcal{L}_{0}$, which defines the measure

$$
\mu_{a b}=\left|\int_{\lambda_{a}}^{\lambda_{b}} \rho(\lambda) d \lambda\right|
$$

\section{EPISTROPHES AND EPISTROPHIC FRACTALS}

\section{A. The Epistrophe Theorem}

A segment of the line of initial conditions $\mathcal{L}_{0}$ that escapes the complex in $k$ iterates lies in the intersection of $\mathcal{L}_{0}$ with the escape zone $E_{-k}$. It is, in fact, one connected component of this intersection. With rare exceptions, the end points of an escape segment are therefore points on the stable manifold $\mathcal{S}{ }^{45}$ We will prove that upon any (transverse) intersection between $\mathcal{S}$ and $\mathcal{L}_{0}$, there converges an epistrophe of escape segments.

The existence of epistrophes is suggested by the following argument. In a small neighborhood of $\mathbf{z}_{\mathbf{X}}$, the map $\mathcal{M}$ is almost linear and can be re-expressed in new canonical coordinates $(Q(q, p), P(q, p))$ by $(Q, P) \mapsto\left(Q^{\prime}, P^{\prime}\right)$, where ${ }^{46}$

$$
\begin{aligned}
& Q^{\prime}=\alpha Q+O(2), \\
& P^{\prime}=(1 / \alpha) P+O(2) .
\end{aligned}
$$

Ignoring the higher order terms, $\mathcal{S}$ and $\mathcal{U}$ are, respectively, the positive $P$ axis and negative $Q$ axis. Suppose $\mathcal{L}_{0}$ is a horizontal line intersecting the positive $P$ axis near $\mathbf{z}_{\mathbf{X}}$, as shown in Fig. 4. Then, again ignoring higher order terms, the width $u\left(E_{-k}\right)$ of the base of an escape zone decreases by a factor of $\alpha$ on each backward iterate. Thus, as the escape zone is mapped backward, it is squeezed by $1 / \alpha$ in the $Q$ direction and stretched by $\alpha$ in the $P$ direction. Clearly, these lobes must eventually intersect $\mathcal{L}_{0}$, and their intersections $\boldsymbol{\epsilon}_{k}$ must form an infinite sequence of geometrically decreasing intervals, converging upon the intersection $\mathbf{z}_{\mathcal{S}}$ of $\mathcal{L}_{0}$ with $\mathcal{S}$; the Euclidean lengths $\lambda_{k}$ decrease as $\lambda_{k+1}=\lambda_{k} / \alpha$ (in the limit $k \rightarrow \infty)$.

The Epistrophe Theorem asserts that such geometric sequences appear for any map with a homoclinic tangle (satisfying Assertions 1-5) and for any differentiable curve $\mathcal{L}_{0}$ intersecting the stable manifold $\mathcal{S}$ at any distance from $\mathbf{z}_{\mathrm{X}}$, even far from the region where the linearization (7) is sensible.

Theorem 1 (Epistrophe Theorem): Let $\mathcal{M}$ be any "saddle-center map" (as defined by Assumptions 1-5) and $\mathbf{z}_{\mathcal{S}}$ be any transverse intersection between the stable manifold $\mathcal{S}$ and the differentiable curve of initial conditions $\mathcal{L}_{0}$. For each $k>0$ choose the escape segment $\epsilon_{k} \subset \mathcal{L}_{0} \cap E_{-k}$ closest to $\mathbf{z}_{\mathcal{S}}$ (as measured along $\mathcal{L}_{0}$.) Then there is some initial $k_{0}$ such that for all $k \geqslant k_{0}$, the escape segment $\epsilon_{k}$ exists and:

(i) The segments $\epsilon_{k}$ converge monotonically upon $\mathbf{z}_{\mathcal{S}}$ (i.e., the distance between $\epsilon_{k}$ and $\mathbf{z}_{\mathcal{S}}$ decreases monotonically).

(ii) Define: $\mu_{k}$-the measure of $\epsilon_{k}$ [using Eq. (6)]; $\gamma_{k}$-the measure between $\epsilon_{k}$ and $\epsilon_{k+1} ; \delta_{k}$-the measure between $\epsilon_{k}$ and $\mathbf{z}_{\mathcal{S}}$. Provided $\rho\left(\mathbf{z}_{\mathcal{S}}\right) \neq 0$, all three measures converge geometrically to zero as 


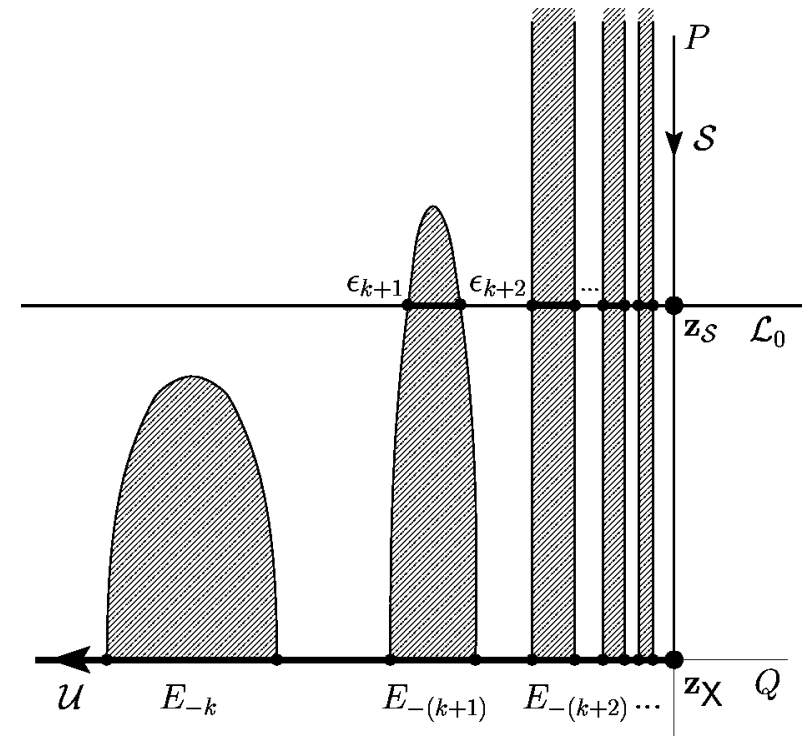

FIG. 4. Introducing new canonical coordinates $(Q, P)$, the saddle-center map $\mathcal{M}$, linearized about the $\mathrm{X}$ point $\mathbf{z}_{\mathrm{X}}$, has the simple form $Q \mapsto \alpha Q$, $P \mapsto(1 / \alpha) P$. The stable manifold $\mathcal{S}$ and unstable manifold $\mathcal{U}$ coincide, respectively, with the positive $P$ and negative $Q$ axes. A sequence of backward iterates of the escape zone $E_{-k}$ is shown shaded. Under each iterate, the width of the lobe decreases by $1 / \alpha$ while the height increases by $\alpha$. Eventually, these iterates must intersect the line of initial conditions $\mathcal{L}_{0}$, shown as a horizontal line passing through the $P$ axis. The escape segments $\epsilon_{k+1}, \epsilon_{k+2}, \ldots$ which are created by these intersections decrease in size by the same geometric factor $\alpha$ (in the asymptotic limit). They eventually converge upon the intersection $\mathbf{z}_{\mathcal{S}}$ of $\mathcal{L}_{0}$ with $\mathcal{S}$.

$$
\begin{aligned}
& \lim _{k \rightarrow \infty} \mu_{k} \alpha^{k}=K_{\mu}>0, \\
& \lim _{k \rightarrow \infty} \gamma_{k} \alpha^{k}=K_{\gamma}>0, \\
& \lim _{k \rightarrow \infty} \delta_{k} \alpha^{k}=K_{\delta}>0,
\end{aligned}
$$

where $\alpha$ is the Liapunov factor of $\mathbf{z}_{X}$ and $K_{\mu}, K_{\gamma}, K_{\delta}$ are positive real numbers. Furthermore,

$$
\lim _{k \rightarrow \infty} \frac{\mu_{k}}{\gamma_{k}}=\frac{K_{\mu}}{K_{\gamma}} \equiv \chi>0
$$

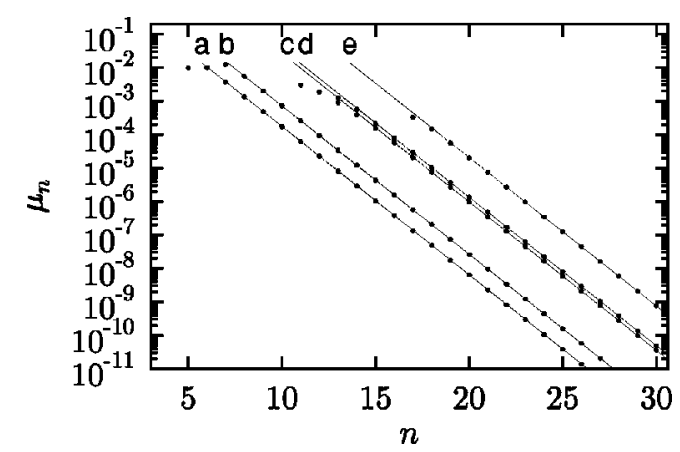

FIG. 5. The length $\mu_{n}$ of an escape segment is plotted as a function of iterate number $n=n_{i}$ for the five epistrophes a-e shown in Fig. 2. The lines all have the same slope, equal to $-\log \alpha$, where $\alpha=2.776$ is the Liapunov factor of the $X$ point in Fig. 1. This factor can be computed analytically from Eqs. (A4) and (A5). Clearly as $n \rightarrow \infty, \mu_{n}$ decays geometrically with $\mu_{n}$ $\rightarrow$ (constant) $\times \alpha^{-n}$.

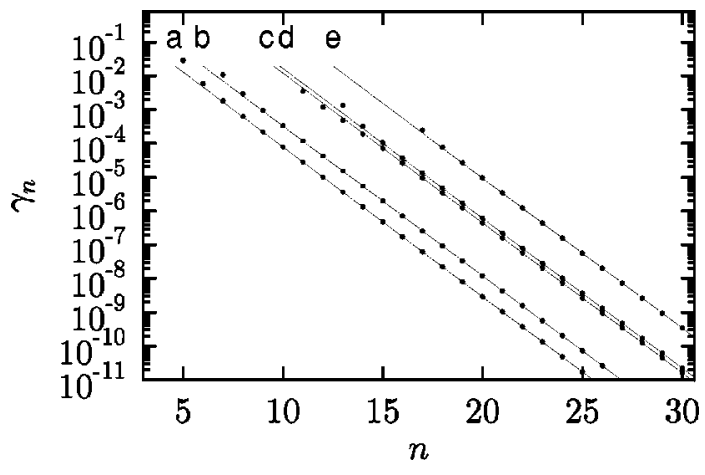

FIG. 6. Analogous to Fig. 5, the distance $\gamma_{n}$ between successive escape segments is plotted as a function of iterate number $n=n_{i}$. It is again readily apparent that as $n \rightarrow \infty, \gamma_{n} \rightarrow$ (constant) $\times \alpha^{-n}$, where $\alpha=2.776$ is the same as in Fig. 5.

$$
\lim _{k \rightarrow \infty} \frac{\mu_{k}}{\delta_{k}}=\frac{K_{\mu}}{K_{\delta}} \equiv \phi>0,
$$

where $\chi=u\left(E_{0}\right) / u\left(C_{0}\right) \quad$ and $\quad \phi=u\left(E_{0}\right) / u\left(\mathbf{P}_{0}\right)=\chi(\alpha$ $-1) /(\alpha+\chi)$. [The lengths $u\left(\mathbf{P}_{0}\right), u\left(E_{0}\right)$ and $u\left(C_{0}\right)$ are defined in Eqs. (3), (5a), and (5c).]

The constants $K_{\mu}, K_{\gamma}, K_{\delta}$ depend upon which epistrophe is examined, but their ratios do not, as evident from the formulas for $\chi$ and $\phi$. This result was unexpected to us; we were struck by the fact that not only the same factor $\alpha$ but also the same ratios $\chi$ and $\phi$ apply to each epistrophe, no matter how far it is from $\mathbf{z}_{X}$.

The Epistrophe Theorem describes the tails of sequences. The value of $k_{0}$ and the exact values of $\mu_{k}, \gamma_{k}$, and $\delta_{k}$ (especially the early values) cannot be predicted from the present considerations. What can be predicted is how $\mu_{k}$, $\gamma_{k}$, and $\delta_{k}$ decay in the asymptotic limit.

The Epistrophe Theorem is proved in Appendix B. Equations (8a), (8b), and (9a) are verified numerically in Figs. 5, 6 , and 7. These plots indicate that the asymptotic behavior predicted by the theorem is approached quickly.

Notice that no epistrophe converges upon the boundary of a stable zone. For example, in the upper half of Fig. 2, epistrophe a converges upward to the boundary of the complex, but there is no epistrophe converging downward where the boundary of the stable zone lies. Similarly, in the bottom

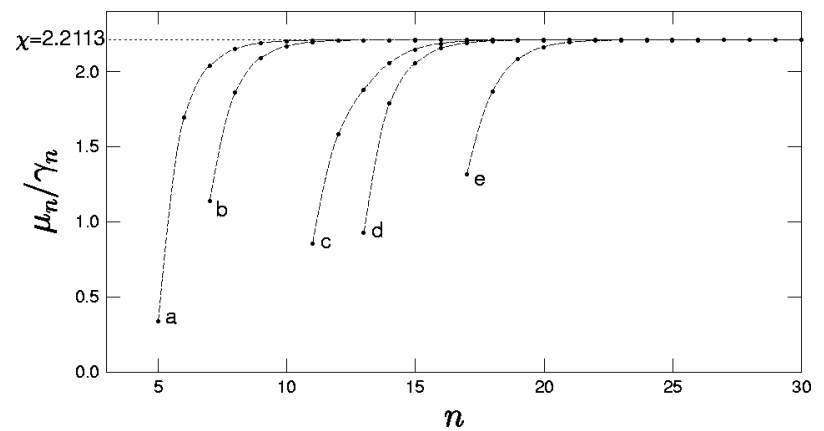

FIG. 7. The ratio $\mu_{n} / \gamma_{n}$ of the data in Fig. 5 to the data in Fig. 6 is plotted. The ratio for each epistrophe $\mathbf{a}-\mathbf{e}$ has the same asymptotic value $\chi$ $=2.2113$, which agrees with an independent computation of $\chi$ from the formula $\chi=u\left(E_{0}\right) / u\left(C_{0}\right)=\lim _{k \rightarrow \infty}\left|\mathbf{Q}_{-k}-\mathbf{P}_{-k}\right| /\left|\mathbf{P}_{-k}-\mathbf{Q}_{-(k+1)}\right|$. 
half of Fig. 2, epistrophe b converges downward to the boundary of the complex, but no epistrophe converges upward to the stable zone.

\section{B. Epistrophes and strophes}

Any orderly infinite sequence of escape segments predicted by the Epistrophe Theorem is called an epistrophe. The irregular beginning of such a sequence, or any escape segment or group of segments that is not part of an epistrophe, is called a strophe. The strophes contain the unpredicted behavior in the escape-time plot.

It is helpful to examine the origin and meaning of the word "epistrophe" because the parts of this word describe the structure of epistrophic fractals. One dictionary ${ }^{47}$ defines "strophe" simply as a stanza of a poem or ballad, while another ${ }^{48}$ defines it as a stanza that might have irregular structure, such as variable length and rhythm. This ambiguity is useful to us, because we might or might not find regular structure in the escape segments that we call strophes.

"Epi-" is used here in the sense of "the end of" or "concluding" (as in epidermis or epilogue). An epistrophe in rhetoric is a repeated ending following a variable beginning. One of the most familiar in American English is from Lincoln's Gettysburg Address: "of the people, by the people, and for the people." Here "of," "by," and "for" are the strophes and the repetitions of "the people" are epistrophes. This is an example in which the epistrophes dominate the structure, as the strophes each have one syllable, while the epistrophes have three. Analogous behavior may occur for dynamical epistrophes: if the Liapunov factor $\alpha$ is close to one, the total length of the segments in an epistrophe will tend to dominate over the length in the strophe.

A quite different epistrophic structure is contained in the Hebrew creation recitative, with its description of the first six days. The descriptions of each day (the strophes) vary in length and structure. Each strophe ends with the epistrophe: "And the evening and the morning were the $[n$th $]$ day." $n$ $=1, \ldots, 6$. The epistrophe is short, and the strophes dominate the length of the narrative. This tends to happen in dynamical epistrophes if the Liapunov factor $\alpha$ is large.

Actually the creation recitative might be called a doubly epistrophic narrative, because it contains seven repetitions of a different epistrophe: "... and God saw that it was good." These are interspersed with a rhythmic structure different from that of the " $n$th day" epistrophe; the second day does not contain this epistrophe, but it appears in other days once or twice, sometimes at the end of the day and sometimes in the middle. Complicated interleaving of two or more different families of epistrophes (typically with different $\alpha$ ) can occur, for example, in a dynamical system if the boundary of the complex contains more than one $\mathrm{X}$ point.

For dynamical applications, we define an "epistrophe" as an infinite sequence of escape segments having the properties described by the Epistrophe Theorem; consistent with rhetoric, an epistrophe has a predictable ending. We define "strophe" less precisely, consistent with its use in rhetoric, and apply it in two contexts: (1) A strophe is the unpredicted beginning of an epistrophe; that is, the first few escape seg- ments $\epsilon_{k_{0}}, \epsilon_{k_{0}+1}, \ldots$ of an epistrophe (with unpredicted values of $k_{0}, \mu_{k_{0}}$, etc.) constitute one strophe. In the following paper, we will show that there is partial predictability of these strophes. (See also Secs. III C and IV.) (2) A strophe is any additional escape segment, or group of escape segments, that is not part of an epistrophe. Several such strophes were indicated by asterisks in Fig. 2. For now, we deliberately avoid giving the word "strophe" a sharp definition; we leave open the possibility that the strophes might later be described in some more complex framework.

\section{Epistrophic self-similarity}

The Epistrophe Theorem implies a certain recursive structure to the escape-time plot: every epistrophe is asymptotically self-similar and is asymptotically related to any other epistrophe by a change in scale. In this sense, there is a kind of asymptotic self-similarity of sequences of escape segments in the escape-time plot. A second type of regularity will be established in the next paper, where we will state and prove an "Epistrophe Start Rule." As a result of the global topology of the tangle, there is a "minimal set" of escape segments. In this minimal set, an epistrophe begins at an iterate $k_{0}$, exactly $\Delta=D+1$ iterates after the segment upon which it converges. (We will explain that the parameter $D$ is the minimal delay time of the tangle.) Hence, in the minimal set there is a simple recursive pattern to the escape segments: on each side of a given escape segment, a new epistrophe begins $\Delta$ iterates later, and these epistrophes converge to the given escape segment as described by the Epistrophe Theorem; then on each side of each segment in the new epistrophes, a further new epistrophe begins $\Delta$ iterates later; at every level, every segment spawns two new epistrophes, ad infinitum. One might expect this to produce a regular selfsimilar (or at least asymptotically self-similar) fractal structure. Indeed, this is readily seen in the "standard" Smale horseshoe (e.g., Ref. 38).

Strict self-similarity and asymptotic self-similarity are consistent with the Epistrophe Theorem and the Epistrophe Start Rule, but they are not guaranteed by these results. First, these results allow the beginnings of the epistrophes to contain irregular lengths that do not follow any simple pattern. Indeed in our numerical studies we do not see any simple pattern to the lengths of the first segment of an epistrophe. More importantly, we find additional, unpredicted strophe segments which are not part of the minimal set, and which therefore do not fit the pattern of the minimal set. Furthermore, numerical evidence seems to indicate that these strophe segments tend to dominate at long times.

It is possible, and even likely, that the strophes obey some higher-order and more complex recursive rules. If one could uncover these rules, one might hope to find a deeper and more complex kind of asymptotic self-similarity in the escape-time plot; one might hope that there would be a finite number of such rules. However, it is generally acknowledged $^{28,29,35,44}$ that to describe the topology of a tangle requires a countable infinity of topological parameters, reflecting the growing topological complexity of the tangle on finer and finer scales. This situation is nicely de- 
scribed by Rückerl and Jung. ${ }^{35}$ Thus, for any finite description of the tangle, one expects eventually to discover in the escape-time plot additional structure which had not yet been predicted and which eventually comes to dominate the structure that is predicted.

We use the term "epistrophic self-similarity" to describe the above-mentioned situation: throughout the escape-time plot and on all scales there are epistrophes; they are all asymptotically self-similar and each is similar to every other. However, there may also be unpredicted strophe segments which also occur on all scales and which may come to dominate the regular epistrophe structure.

What is the distinction between asymptotic and epistrophic self-similarity? A fractal has asymptotic selfsimilarity if through repeated magnifications about any point of the fractal, the pattern converges to an asymptotic structure. Epistrophic self-similarity is weaker, requiring only the existence of epistrophes, as described earlier. If when repeatedly magnifying the fractal, one continues to see new structures emerge, then the fractal certainly does not possess asymptotic self-similarity, but may still possess epistrophic self-similarity.

\section{Epistrophic fractals}

Thus far, we have focused on the segments of $\mathcal{L}_{0}$ that escape the complex. The question remains: How do the epistrophes influence the points that survive and never escape? Jung and Scholz observed that the stable manifolds of all the bound orbits (periodic and aperiodic) give rise to a Cantor set of singularities for a scattering function. ${ }^{34}$ We show that the Epistrophe Theorem itself directly leads to a Cantor set within the set of surviving points.

Obviously any point inside a stable island survives. Accordingly, we define the stable domain $S$ to be the union of all open disks each of which contains no escaping points. The set $S$ is an open set in the plane, containing the interiors of all the stable islands. Any point in $S$ is stably surviving, in the sense that any sufficiently small displacement of the point still results in survival. (By definition, $S$ contains all stably surviving points.) Continuity guarantees that points on the boundary of $S$ (the "shoreline" of the stable islands) also survive. However, they are unstably surviving in the sense that a small perturbation can cause them to escape.

There are other sets of unstably surviving points in the complex besides the shoreline of the islands of stability. (1) The $X$ point $\mathbf{z}_{X}$ is unstably surviving. (2) Many unstable periodic orbits are embedded in the chaotic sea surrounding the islands of stability. (3) There may be entire curves of neutrally stable periodic orbits. (4) Each unstable periodic orbit has stable manifolds which do not escape. (5) There may be Cantori of surviving points in the chaotic sea; a Cantorus is an invariant Cantor set which is the remnant of a dissolved KAM torus. ${ }^{27}$ (6) There are chaotic trajectories that wander about in the chaotic sea but never escape. There might be other types of unstably surviving points which we have omitted here.

The line of initial conditions $\mathcal{L}_{0}$ runs through the complex and may intersect some of the above-mentioned sets of

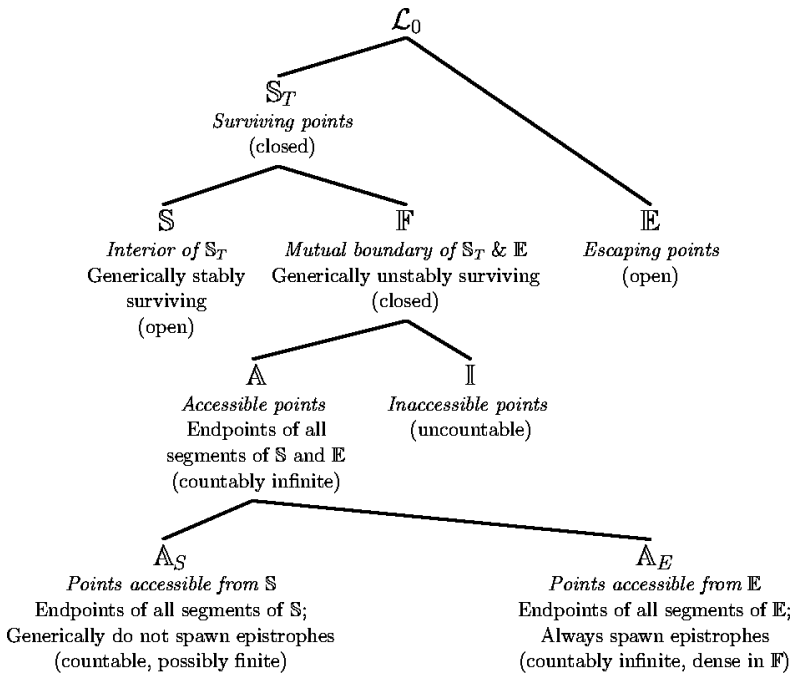

FIG. 8. The above schematic illustrates the relations between various subsets of $\mathcal{L}_{0}$ which are important for understanding the fractal nature of escape. Each branching of the tree represents a partition of the upper set into the two lower disjoint subsets.

surviving points, while missing others. Certainly, $\mathcal{L}_{0}$ intersects the stable manifold of $\mathbf{z}_{X}$ an infinite number of times. We also expect that $\mathcal{L}_{0}$ may intersect the set $S$ of stable islands. Each such intersection will produce an open segment of $\mathcal{L}_{0}$ which does not escape, bounded by surviving end points which are part of the shoreline of $S$. These are the only generic intervals of $\mathcal{L}_{0}$ known to us that do not escape. ${ }^{49}$

The Epistrophe Theorem yields specific results about the structure of the set $\mathrm{S}_{T}$ of surviving points of $\mathcal{L}_{0}$. The set of escaping points is denoted $\mathbb{E}$. (See Fig. 8.) Since each escape segment is open, the set $\mathbb{E}$ is also open. ${ }^{50}$ The set $\mathrm{S}_{T}$, being everything not in $\mathbb{E}$, is closed (and hence compact).

We define $S$ to be the interior of the set $S_{T}$; that is, $S$ is the union of all open sets in $S_{T}$. As noted earlier, generically $S$ consists entirely of points in the interior of stable islands. Any surviving point not in $\mathrm{S}$ we put in a set denoted $\mathbb{F}$, so $\mathbb{F}$ contains whatever points of $\mathcal{L}_{0}$ survive, other than open intervals; all points in $\mathbb{F}$ are unstably surviving. The set $\mathbb{F}$ is constructed by the following process. After a finite number $n$ of iterates of the map, a finite number of escape segments will have been removed (assuming that $\mathcal{L}_{0}$ is analytic). Between these escape segments are intervals that have managed to survive for $n$ iterates. As we continue to iterate the map, we remove subintervals of these surviving intervals. In the limit, if there are any surviving intervals, we put them into the set $\mathrm{S}$. What remains is $\mathbb{F}$. This is very much like the construction of the Cantor middle-third set. In fact, we demonstrate in the following that $\mathbb{F}$ is a topological Cantor set.

A topological Cantor set is any set which is homeomorphic to a subset of the real line and which is compact (i.e., closed and bounded), perfect (i.e., containing no isolated points), and totally disconnected (i.e., containing no intervals). ${ }^{51}$ All Cantor sets are homeomorphic (topologically equivalent) to each other, ${ }^{51}$ so when thinking about the topology of Cantor sets, it is sufficient to imagine the middlethird Cantor set. Cantor sets may differ in their metric struc- 
ture, that is, in the lengths and separations of the segments that are deleted to form the set, and different metric structures will in general result in different fractal dimensions of the set.

We assume in the following that $\mathcal{L}_{0}$ is nowhere tangent to the stable manifold. (The results can be modified to account for such tangencies, but it would obfuscate our discussion.) The set $\mathbb{F}$ is a Cantor set because it satisfies the three necessary requirements. (1) It is closed and bounded, i.e., compact (by its definition). (2) It is totally disconnected, i.e., contains no intervals (because they have been explicitly excluded). (3) It is perfect, i.e., contains no isolated points. The third assertion follows from the Epistrophe Theorem. If there were an isolated surviving point, then directly on its right there would have to be a (connected) open interval of escaping points. But any such interval must lie inside a single escape segment. Hence, the isolated point must be the left end point of that escape segment and an intersection between $\mathcal{L}_{0}$ and $\mathcal{S}$ (which is transverse by assumption). Therefore, the point must have an epistrophe converging upon its left side, which implies that the point is in fact not isolated.

The fractal $\mathbb{F}$ is the mutual boundary of the sets $S_{T}$ and $\mathbb{E}$. Topologically, the boundary of a set $U$ is the closure of $U$ (i.e., the smallest closed set containing $U$ ) minus the interior of $U$ (i.e., the largest open set contained in $U$ ). By definition, $\mathbb{F}$ is the boundary of $S_{T}$. We see that $\mathbb{F}$ is the boundary of $\mathbb{E}$ because any point in $\mathbb{F}$ must have a point in $\mathbb{E}$ arbitrarily close to it, otherwise it would be in the interior $\mathrm{S}$ of $\mathrm{S}_{T}$. We can now summarize the situation as follows: inside the complex, the line of initial conditions is partitioned into three sets: (1) the set $\mathrm{S}$ of all open surviving intervals, (2) the set $\mathbb{E}$ of all open escaping intervals, and (3) their mutual boundary $\mathbb{F}$; this boundary is a Cantor set.

Like all Cantor sets, $\mathrm{F}$ can be divided into two subsets consisting of the accessible and inaccessible points. The fractal $\mathbb{F}$ is a line segment with a countable number of open intervals removed, some being escape segments of $\mathbb{E}$ and some being (generically) interiors of islands in S. An end point of an escape segment is said to be accessible from $\mathbb{E}$ because there exists a path beginning in $\mathbb{E}$ and terminating on the end point without encountering any other point outside of $\mathbb{E}$; we denote the countably infinite set of points accessible from $\mathbb{E}$ by $A_{E}$. Similarly an end point of a segment in $S$ is said to be accessible from $\mathrm{S}$, and we denote the set of such points by $A_{S}$. The set $A_{S}$ may be countably infinite, finite, or empty. Together $A_{E}$ and $A_{S}$ form the countably infinite set $A=A_{E} \cup A_{S}$ of accessible points of $\mathbb{F}$ (i.e., the set of all end points of deleted intervals, whether in $\mathbb{E}$ or $S$ ). Since the fractal $\mathbb{F}$ is uncountably infinite, most points in $\mathbb{F}$ lie in the set, denoted $\mathbb{I}$, of inaccessible points.

Every point of $A_{E}$ lies on the stable manifold and hence has an epistrophe converging upon it. However, the points of $A_{S}$ generically do not lie on the stable manifold, but are rather part of the shoreline of $S$. So we do not expect epistrophes to converge upon the end points of a surviving segment of $\mathcal{L}_{0}$. (This is readily apparent in Fig. 2.) Nevertheless, the epistrophes are dense in the fractal, or more precisely, the set $A_{E}$ is dense in $\mathbb{F}$.

Because the escape segments exhibit what we called epistrophic self-similarity in Sec. III C and because $\mathbb{F}$ is a Cantor set, we call $\mathbb{F}$ an epistrophic fractal.

\section{DISCUSSION AND FUTURE RESEARCH}

The Epistrophe Theorem predicts the existence of an epistrophe converging geometrically upon the end point of any escape segment; it also characterizes the asymptotic behavior of the convergence in terms of geometric quantities $\alpha$ and $\chi$. However, none of our present results say anything about how an epistrophe begins. Most notably, we do not predict the iterate at which an epistrophe starts, and we certainly do not estimate the lengths or separation distances of the early escape segments.

To understand the early behavior of an epistrophe, we must look at the global topology of the tangle, which is the topic of the following paper. In that paper, we prove that another pattern appears in the escape-time plots: the first segment $\epsilon_{k+\Delta}$ of an epistrophe is spawned at some number of iterates $\Delta$ later than the segment $\epsilon_{k}^{\prime}$ upon which the epistrophe converges. (This fact was observed by Tiyapan and Jaffe $^{-5}$ and by Jung and co-workers ${ }^{35-37}$ for certain lines of initial conditions in scattering problems.) This pattern is clearly visible in Fig. 2 with $\Delta=6$. The pattern follows from the fact that the topological structure of the map forces the existence of a certain minimal required set of escape segments; this minimal set can be shown to have the stated recursive pattern, for $n_{i}$ sufficiently large. Typically there are additional escape segments (strophes) not predicted by the pattern; some of these unpredicted segments are marked by asterisks in Fig. 2. Nevertheless, the minimal set seems to characterize the early structure of the escape-time plots remarkably well. However, based on numerical evidence, we believe that for large enough iterate number, the minimal set will eventually be overwhelmed by the unpredicted segments, both in total number at a given iterate and in total measure. (Notice that the unpredicted segments in Fig. 2 tend to be the longer segments at high iterate number.)

In future papers, we shall also present theorems concerning the winding number (Fig. 2), and we shall show how all these concepts describe the ionization of a hydrogen atom placed in external electric and magnetic fields.

We conclude by noting that standard references on fractals $^{52}$ discuss at least three distinct types of selfsimilarity: (1) regular self-similarity, as for the Cantor middle-third set or the Koch snowflake; (2) asymptotic selfsimilarity, which characterizes, for example, sequences of period-doubling bifurcations; (3) statistical self-similarity, which might describe coastlines or clouds. To this list we add "epistrophic self-similarity," in which at all levels of resolution there are asymptotically similar sequences, but additional unpredicted segments may also persist.

\section{ACKNOWLEDGMENTS}

The authors would like to thank Professor Nahum Zobin for many useful discussions. This work was financially supported by the National Science Foundation. 


\section{APPENDIX A: A FAMILY OF SADDLE-CENTER MAPS}

We define a map $\mathcal{M}\left(q_{1}, p_{1}\right)=\left(q_{2}, p_{2}\right)$ by

$$
\begin{aligned}
& q_{2}=q_{1}+\frac{\partial G(\bar{q}, \bar{p})}{\partial \bar{q}}, \\
& p_{2}=p_{1}-\frac{\partial G(\bar{q}, \bar{p})}{\partial \bar{q}}, \\
& \bar{q}=\left(q_{1}+q_{2}\right) / 2, \\
& \bar{p}=\left(p_{1}+p_{2}\right) / 2,
\end{aligned}
$$

where the "Poincaré generator" $G(q, p)$ is ${ }^{53,54}$

$$
G(q, p)=\tau\left[\frac{p^{2}}{2 m}+V(q)\right]
$$

and where

$$
V(q)=-\operatorname{sech}(q)-f q
$$

is a local potential well that goes to infinity on the left but only has a potential barrier on the right. It can be shown that this map is canonical and that it is well defined on the entire phase plane.

A fixed point of the map corresponds to a stationary point of $G(q, p)$, where $\partial G / \partial p=\partial G / \partial q=0$. The fixed point is stable or unstable according to whether the Hessian determinant $D=\left(\partial^{2} G / \partial p^{2}\right) \partial^{2} G / \partial q^{2}-\left(\partial^{2} G / \partial q \partial p\right)^{2}$ is positive or negative ${ }^{53,54}$ Accordingly, the map (A1) has exactly one stable and one unstable fixed point, located at the local minimum and the local maximum of $V(q)$. The eigenvalues of a fixed point are given by

$$
\alpha_{ \pm}=\frac{2 \pm \sqrt{-D}}{2 \mp \sqrt{-D}} .
$$

For the unstable fixed point of the map (A1), we have the explicit formula

$$
\begin{aligned}
& D=-\frac{\tau^{2}}{m} \sqrt{\frac{J(1-\sqrt{J})}{2},} \\
& J=1-4 f^{2},
\end{aligned}
$$

from which the Liapunov factor $\alpha=\alpha_{+}>1$ can be computed.

\section{APPENDIX B: PROOF OF THE EPISTROPHE THEOREM}

\section{Normal form near an $\mathrm{X}$ point}

We will need the following "normal form" theorem proved by Moser. ${ }^{55}$

Theorem 2 (Moser, Ref. 55): In the neighborhood of an $\mathrm{X}$ point of an analytic area- and orientation-preserving map of the plane $(q, p) \mapsto\left(q^{\prime}, p^{\prime}\right)$, there exists an analytic areapreserving change of coordinates $(q, p) \mapsto(Q, P)$ that places the map into the normal form $(Q, P) \mapsto\left(Q^{\prime}, P^{\prime}\right)$ satisfying

$$
\begin{aligned}
& Q^{\prime}=Q[\alpha+f(Q P)], \\
& P^{\prime}=P /[\alpha+f(Q P)],
\end{aligned}
$$

where $\alpha$ is the Liapunov factor of the $\mathrm{X}$ point. The coordinate change and the function $f(Q P)$ are power series that have a nonvanishing radius of convergence about the $\mathrm{X}$ point. The function $f$ depends only on the product $Q$ times $P$, and $f(0)=0$.

A corollary of Moser's theorem is that the mapping (B1) preserves the product

$$
Q^{\prime} P^{\prime}=Q P,
$$

so the hyperbolic curves $Q P=$ constant are invariant sets under the map. We choose an open set $D$ that is convex in the $P Q$ coordinates, that contains the $\mathrm{X}$ point of $\mathcal{M}$, and in which the normal form converges.

\section{Convergence factors are invariant under differentiable mappings}

Consider an infinite sequence of points $\mathbf{z}_{n}=\left(q_{n}, p_{n}\right)$ that lie on a differentiable curve $\mathcal{C}$ and that converge geometrically to a point $\mathbf{z}_{\infty} \in \mathcal{C}$; i.e., the Euclidean arclength $d_{n}$, measured along $\mathcal{C}$ between $\mathbf{z}_{n}$ and $\mathbf{z}_{\infty}$, satisfies

$$
\lim _{n \rightarrow \infty} d_{n} \beta^{n}=K,
$$

for some $\beta>1$ and $K>0$. We call $\beta$ the convergence factor. For any map of the plane $\mathcal{N}$, which is differentiable and locally invertible about $\mathbf{z}_{\infty}$, define $D_{n}$ as the Euclidean arclength between $\mathbf{Z}_{n}=\mathcal{N}\left(\mathbf{z}_{n}\right)$ and $\mathbf{Z}_{\infty}=\mathcal{N}\left(\mathbf{z}_{\infty}\right)$, measured along $\mathcal{N}(\mathcal{C})$.

Lemma 1: The convergence factor $\beta$ is invariant under the mapping $\mathcal{N}$, that is,

$$
\lim _{n \rightarrow \infty} D_{n} \beta^{n}=J_{0} K>0 \text {. }
$$

Here, $J_{0}=|J \hat{\mathbf{t}}|$ where $J=\partial \mathcal{N} /\left.\partial \mathbf{z}\right|_{\mathbf{z}_{\infty}}$ is the $2 \times 2$ Jacobian matrix evaluated at $\mathbf{z}=\mathbf{z}_{\infty}$ and $\hat{\mathbf{t}}$ is the unit tangent to the curve $\mathcal{C}$ at $\mathbf{z}_{\infty}$.

The notation || denotes the standard Euclidean vector norm. The proof of this lemma is a simple exercise.

\section{Iterates of a curve intersecting the stable manifold approach the unstable manifold}

Consider any differentiable curve $\mathcal{C}_{0}$ having a transverse intersection with the stable manifold $\mathcal{S}$ of $\mathcal{M}$ at a point $\mathbf{r}_{0}$ $=\left(q_{0}, p_{0}\right)$ having canonical length coordinate $r_{0}=s\left(\mathbf{r}_{0}\right)$ along $\mathcal{S}$; we assume $\mathcal{C}_{0}$ does not intersect the segment of $\mathcal{S}$ joining $\mathbf{z}_{\mathbf{X}}$ to $\mathbf{r}_{0}$ (Fig. 9). The differentiable curve $\mathcal{C}_{1}$ $=\mathcal{M}\left(\mathcal{C}_{0}\right)$ intersects $\mathcal{S}$ at a point $\mathbf{r}_{1}=\left(q_{1}, p_{1}\right)=\mathcal{M}\left(\mathbf{r}_{0}\right)$, which is closer to the $X$ point $\mathbf{z}_{X}$. By Eq. (2) $\mathbf{r}_{1}$ has canonical length coordinate $r_{1}=s\left(\mathbf{r}_{1}\right)=r_{0} / \alpha$. Applying $\mathcal{M}$ repeatedly, we obtain an infinite sequence of curves $\mathcal{C}_{n}$ that intersect $\mathcal{S}$ in a sequence $\mathbf{r}_{n}$ converging geometrically to $\mathbf{z}_{\mathbf{X}}$ with convergence factor equal to the Liapunov factor $\alpha: r_{n} \alpha^{n}=r_{0} \neq 0$.

The curves $\mathcal{C}_{n}$ "approach the unstable manifold" in the sense that every point $\mathbf{z}_{\mathcal{U}}$ on $\mathcal{U}$ is the limit of a sequence of points $\mathbf{z}_{n} \in \mathcal{C}_{n}$ converging geometrically with factor $\alpha$.

Lemma 2: For any differentiable curve $\mathcal{C}_{0}$ passing transversely through $\mathcal{S}$ at a point $\mathbf{r}_{0}$ and for any differentiable curve $\overline{\mathcal{C}}$ passing transversely through $\mathcal{U}$ at a point $\mathbf{z}_{\mathcal{U}}$, the 


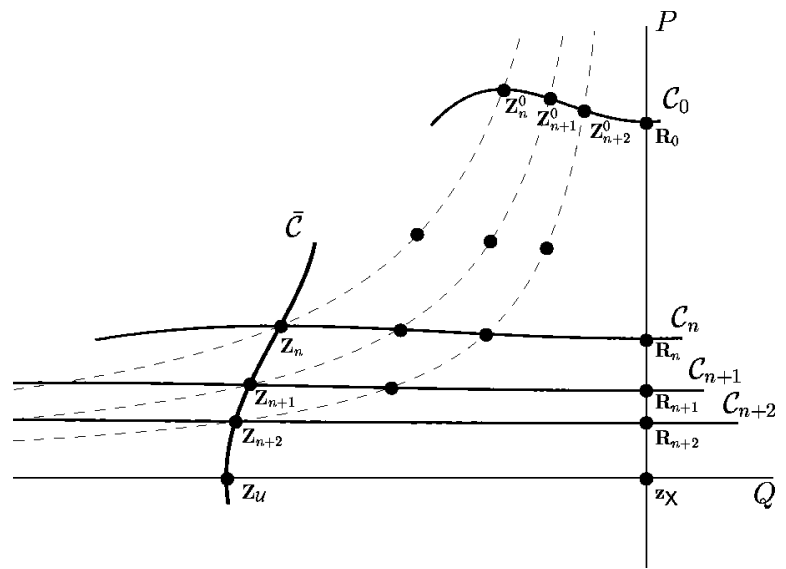

FIG. 9. This diagram, used for proving Lemma 2, depicts the neighborhood of $\mathbf{z}_{\mathrm{X}}$ (the origin) in normal-form coordinates $(Q, P)$. The dashed lines are the invariant hyperbolas of the map. The curves $\mathcal{C}_{n}, \mathcal{C}_{n+1}$, and $\mathcal{C}_{n+2}$ are the $n$ th, $(n+1)$ th, and $(n+2)$ th iterates of $\mathcal{C}_{0}$, respectively. Similarly, the points $\mathbf{Z}_{n}, \mathbf{Z}_{n+1}$, and $\mathbf{Z}_{n+2}$ lying on $\overline{\mathcal{C}}$ are the $n$ th, $(n+1)$ th, and $(n$ +2 )th iterates of the points $\mathbf{Z}_{n}^{0}, \mathbf{Z}_{n+1}^{0}$, and $\mathbf{Z}_{n+2}^{0}$, respectively. The unlabeled dots represent intermediate iterates of these points.

curves $\mathcal{C}_{n}=\mathcal{M}^{n}\left(\mathcal{C}_{0}\right)$ and $\overline{\mathcal{C}}$ intersect for all $n$ large enough. We further assume $\mathcal{C}_{0}$ does not intersect the segment of $\mathcal{S}$ joining $\mathbf{z}_{\mathrm{X}}$ to $\mathbf{r}_{0}$. We then choose $\mathbf{z}_{n}$ to be the point in $\mathcal{C}_{n} \cap \overline{\mathcal{C}}$ closest to $\mathbf{z}_{\mathcal{U}}$ (as measured along $\overline{\mathcal{C}}$ ). The sequence $\mathbf{z}_{n}$ satisfies

$$
\begin{aligned}
& \lim _{n \rightarrow \infty} \mathbf{z}_{n}=\mathbf{z}_{\mathcal{U}}, \\
& \lim _{n \rightarrow \infty}\left|\mathbf{z}_{n}-\mathbf{z}_{\mathcal{U}}\right| \alpha^{n}=A\left(\mathbf{z}_{\mathcal{U}}, \hat{\mathbf{t}}\right) r_{0} \neq 0,
\end{aligned}
$$

where $\alpha$ is the Liapunov factor of $\mathbf{z}_{\mathbf{X}}$ and $A\left(\mathbf{z}_{\mathcal{U}}, \hat{\mathbf{t}}\right)$ is a function that, for a given map $\mathcal{M}$, depends only on the intersection point $\mathbf{z}_{\mathcal{U}}$ and the tangent $\hat{\mathbf{t}}$ to the curve $\overline{\mathcal{C}}$ at $\mathbf{z}_{\mathcal{U}}$.

Proof: First suppose $\mathbf{z}_{\mathcal{U}}$ and $\mathbf{r}_{0}$ lie within the domain $D$ in which the normal form applies (Appendix B 1). Within $D$, we use the normal-form coordinates $(Q, P)$, in which case $\mathbf{z}_{\mathcal{U}}$ and $\mathbf{r}_{0}$ are represented by $\mathbf{Z}_{\mathcal{U}}=\left(Q_{\mathcal{U}}, 0\right)$ and $\mathbf{R}_{0}=\left(0, R_{0}\right)$. By Lemma 1 , if we prove Lemma 2 in the $(Q, P)$ coordinates then we have proved Lemma 2 in the original $(q, p)$ coordinates.

The point $\mathbf{Z}_{n}=\left(Q_{n}, P_{n}\right)$ in $\mathcal{C}_{n} \cap \overline{\mathcal{C}}$ is the $n$th iterate of some point $\mathbf{Z}_{n}^{0}=\left(Q_{n}^{0}, P_{n}^{0}\right)$ in $\mathcal{C}_{0}$. (See Fig. 9.) Thus, iterating Eq. (B1), we see that

$$
\begin{aligned}
& Q_{n}=Q_{n}^{0}\left[\alpha+f\left(Q_{n} P_{n}\right)\right]^{n}, \\
& P_{n}=P_{n}^{0}\left[\alpha+f\left(Q_{n} P_{n}\right)\right]^{-n} .
\end{aligned}
$$

Near the point $\mathbf{R}_{0}$ the curve $\mathcal{C}_{0}$ is the graph of a differentiable function $P=C_{0}(Q)$, and similarly, near the point $\mathbf{Z}_{\mathcal{U}}$, the curve $\overline{\mathcal{C}}$ is the graph of a differentiable function $Q=\bar{C}(P)$. Combining this with Eq. (B7), we find that for $n$ large enough $P_{n}$ must satisfy

$$
\begin{aligned}
& \bar{C}\left(P_{n}\right)=Q_{n}^{0}\left[\alpha+f\left(P_{n} \bar{C}\left(P_{n}\right)\right)\right]^{n}, \\
& P_{n}=C_{0}\left(Q_{n}^{0}\right)\left[\alpha+f\left(P_{n} \bar{C}\left(P_{n}\right)\right)\right]^{-n} .
\end{aligned}
$$

Solving for $Q_{n}^{0}$ in Eq. (B8a) and inserting the result into Eq. (B8b), we find

$$
P_{n}=G_{n}\left(P_{n}\right)\left[\alpha+F\left(P_{n}\right)\right]^{-n},
$$

where

$$
\begin{aligned}
& G_{n}(P)=C_{0}\left(\bar{C}(P)[\alpha+F(P)]^{-n}\right), \\
& F(P)=f(P \bar{C}(P)) .
\end{aligned}
$$

The functions $G_{n}(P)$ and $F(P)$ have the following properties: (1) $G_{n}(P)$ and $F(P)$ are well defined, differentiable functions in the neighborhood of $P=0$ (for $n$ sufficiently large); (2) $F(0)=0$; (3) $G_{n}(0) \neq 0$. Observe that Eq. (B9) is an implicit expression for $P_{n}$.

Lemma 2a: For each $n$, choose $P_{n}>0$ to be the smallest real solution to Eq. (B9). Then for $n$ sufficiently large, $P_{n}$ is well defined and $\lim _{n \rightarrow \infty} P_{n} \alpha^{n}=R_{0}$.

Proof: Define $E_{n}(P)=P[\alpha+F(P)]^{n} / G_{n}(P)-1$. Then $P_{n}$ satisfies Eq. (B9) in the neighborhood of 0 if and only if it is a zero of $E_{n}$. Let $\epsilon>0$ be given. Recalling that $F(0)$ $=0$ and $\alpha>1$, we assume that $\epsilon$ is small enough so that $\alpha$ $+F(\epsilon)>1$ and $\bar{C}(\epsilon)$ is well defined. This implies

$$
\lim _{n \rightarrow \infty} G_{n}(\epsilon)=C_{0}(0)=R_{0}>0,
$$

and furthermore,

$$
\lim _{n \rightarrow \infty} E_{n}(\epsilon)=\lim _{n \rightarrow \infty} \frac{\epsilon[\alpha+F(\epsilon)]^{n}}{R_{0}}=+\infty .
$$

Combining this result with the fact that $E_{n}(0)=-1$ [since $\left.G_{n}(0) \neq 0\right]$, we see that there must be a zero of $E_{n}$ between 0 and $\epsilon$ for all $n$ large enough. Since $\epsilon>0$ was arbitrarily small, we can construct a sequence $P_{n}$ of zeros of $E_{n}(P)$ such that $P_{n}$ goes to 0 . We choose the sequence $P_{n}$ to consist of the smallest positive roots of $E_{n}$.

Next, notice that

$$
\begin{aligned}
\lim _{n \rightarrow \infty} n P_{n} & =\lim _{n \rightarrow \infty} n\left[\alpha+F\left(P_{n}\right)\right]^{-n} G_{n}\left(P_{n}\right) \\
& =\lim _{n \rightarrow \infty} n\left[\alpha+F\left(P_{n}\right)\right]^{-n} R_{0}=0,
\end{aligned}
$$

where the first equality follows from Eq. (B9), the second from Eq. (B12), and the last from $F(0)=0$. Finally,

$$
\begin{aligned}
\lim _{n \rightarrow \infty} \ln \left(P_{n} \alpha^{n}\right) & =\ln R_{0}-\lim _{n \rightarrow \infty} n \ln \left[1+F\left(P_{n}\right) / \alpha\right] \\
& =\ln R_{0}-\left.\frac{1}{\alpha} \frac{d F}{d P}\right|_{P=0} \lim _{n \rightarrow \infty} n P_{n}=\ln R_{0},
\end{aligned}
$$

where the first equality follows from Eqs. (B9) and (B12), the second by expanding $F\left(P_{n}\right)$ about $P_{n}=0$, and the last from Eq. (B14). This completes the proof of Lemma 2a.

The sequence $P_{n}$ yields the sequence of points $\mathbf{Z}_{n}$ $=\left(Q_{n}, P_{n}\right)=\left(\bar{C}\left(P_{n}\right), P_{n}\right)$ in the statement of Lemma 2 . Equation (B6) in the normal-form coordinates follows immediately from Lemma $2 \mathrm{a}$, 


$$
\begin{aligned}
\lim _{n \rightarrow \infty}\left|\mathbf{Z}_{n}-\mathbf{Z}_{\mathcal{U}}\right| \alpha^{n} & =\lim _{n \rightarrow \infty}\left[P_{n}^{2}+\left(Q_{n}-Q_{\mathcal{U}}\right)^{2}\right]^{1 / 2} \alpha^{n} \\
& =A(\hat{\mathbf{t}}) R_{0} \neq 0,
\end{aligned}
$$

where $A(\hat{\mathbf{t}})=\left[1+\left(d \bar{C} /\left.d P\right|_{P=0}\right)^{2}\right]^{1 / 2}$ obviously depends only on the tangent $\hat{\mathbf{t}}$ to $\overline{\mathcal{C}}$ at the intersection point $\mathbf{Z}_{\mathcal{U}}=\left(Q_{\mathcal{U}}, 0\right)$. Lemma 1 shows that the transformation back to the original $(q, p)$ coordinates introduces a Jacobian factor (dependent on $\mathbf{z}_{\mathcal{U}}$ and $\left.\hat{\mathbf{t}}\right)$ which can be absorbed into a new $A\left(\mathbf{z}_{\mathcal{U}}, \hat{\mathbf{t}}\right)$.

At this point Lemma 2 is proved provided $\mathbf{z}_{\mathcal{U}}$ and $\mathbf{r}_{0}$ lie within $D$, where the normal form is valid. To extend the theorem to arbitrary $\mathbf{r}_{0}$ on the stable manifold and arbitrary $\mathbf{z}_{\mathcal{U}}$ on the unstable manifold, we use the following "forwardbackward mapping trick."

First suppose $\mathbf{z}_{\mathcal{U}}$ lies within $D$ but $\mathbf{r}_{0}$ lies outside of $D$. We apply $\mathcal{M}$ a finite number of times $k$ until $\mathbf{r}_{k}$ lies within $D$, and then we repeat the preceding argument.

On the other hand, if $\mathbf{z}_{\mathcal{U}}$ lies outside of $D$ we apply the inverse map $\mathcal{M}^{-1}$ to $\mathbf{z}_{\mathcal{U}} j$ times until $\mathbf{z}_{\mathcal{U}}^{-j}=\mathcal{M}^{-j}\left(\mathbf{z}_{\mathcal{U}}\right)$ lies within $D$. Then by the preceding argument, we identify the sequence of points $\mathbf{z}_{n-j}^{-j} \in \mathcal{C}_{n-j} \cap \mathcal{M}^{-j}(\overline{\mathcal{C}})$ converging to $\mathbf{z}_{\mathcal{U}}^{-j}$. We map this sequence forward $j$ times to arrive at the sequence $\mathbf{z}_{n} \in \mathcal{C}_{n} \cap \overline{\mathcal{C}}$ converging to $\mathbf{z}_{\mathcal{U}}$. By Lemma 1 , the convergence factor remains $\alpha$; we also acquire a Jacobian factor $J_{0}$ from the transformation, but it will depend only on $\mathbf{z}_{\mathcal{U}}$ and $\hat{\mathbf{t}}$ and can be absorbed into a new $A\left(\mathbf{z}_{\mathcal{U}}, \hat{\mathbf{t}}\right)$. $\quad \mathcal{Q E D}$

Note that $A\left(\mathbf{z}_{\mathcal{U}}, \hat{\mathbf{t}}\right)$ in Lemma 2 does not depend on the curve $\mathcal{C}_{0}$. The following corollary exploits this fact.

Lemma 3: For any two differentiable curves $\mathcal{C}_{0}$ and $\mathcal{C}_{0}^{\prime}$, each passing transversely through $\mathcal{S}$ at the points $\mathbf{r}_{0}$ and $\mathbf{r}_{0}^{\prime}$ $\neq \mathbf{r}_{0}$, respectively, and for any differentiable curve $\overline{\mathcal{C}}$ passing transversely through $\mathcal{U}$ at a point $\mathbf{z}_{\mathcal{U}}$, the curves $\mathcal{C}_{n}$ $=\mathcal{M}^{n}\left(\mathcal{C}_{0}\right)$ and $\mathcal{C}_{n}^{\prime}=\mathcal{M}^{n}\left(\mathcal{C}_{0}^{\prime}\right)$ both intersect $\overline{\mathcal{C}}$ for all $n$ large enough. We further assume $\mathcal{C}_{0}\left(\right.$ or $\left.\mathcal{C}_{0}^{\prime}\right)$ does not intersect the segment of $\mathcal{S}$ joining $\mathbf{z}_{\mathbf{X}}$ to $\mathbf{r}_{0}\left(\right.$ or $\left.\mathbf{r}_{0}^{\prime}\right)$. We choose $\mathbf{z}_{n}\left(\right.$ or $\left.\mathbf{z}_{n}^{\prime}\right)$ to be the point in $\mathcal{C}_{n} \cap \overline{\mathcal{C}}$ (or $\mathcal{C}_{n}^{\prime} \cap \overline{\mathcal{C}}$ ) closest to $\mathbf{z}_{\mathcal{U}}$ (as measured along $\overline{\mathcal{C}}$.) The sequences $\mathbf{z}_{n}$ and $\mathbf{z}_{n}^{\prime}$ satisfy

$$
\begin{aligned}
& \lim _{n \rightarrow \infty} \mathbf{z}_{n}=\lim _{n \rightarrow \infty} \mathbf{z}_{n}^{\prime}=\mathbf{z}_{\mathcal{U}}, \\
& \lim _{n \rightarrow \infty}\left|\mathbf{z}_{n}-\mathbf{z}_{n}^{\prime}\right| \alpha^{n}=A\left(\mathbf{z}_{\mathcal{U}}, \hat{\mathbf{t}}\right) d_{0} \neq 0,
\end{aligned}
$$

where $d_{0}$ is the canonical length between $\mathbf{r}_{0}$ and $\mathbf{r}_{0}^{\prime}$ (measured along $\mathcal{S}), \alpha$ is the Liapunov factor of $\mathbf{z}_{\mathbf{X}}$, and $A\left(\mathbf{z}_{\mathcal{U}}, \hat{\mathbf{t}}\right)$ is the same function as in Lemma 2.

\section{The Epistrophe Theorem}

We now complete the proof of the Epistrophe Theorem. We cut the $\mathcal{U}$ boundary of the capture zone $C_{1}$ (see Fig. 3) at an arbitrary point $\mathbf{z}_{\mathrm{cut}}$, creating the following two curves: (1) $\mathcal{C}_{\mathbf{Q}_{0}}$ begins at $\mathbf{z}_{\text {cut }}$ and continues backward along $\mathcal{U}$ until just past $\mathbf{Q}_{0}$; (2) $\mathcal{C}_{\mathbf{P}_{1}}$ begins at $\mathbf{z}_{\text {cut }}$ and continues forward along $\mathcal{U}$ until just past $\mathbf{P}_{1}$. Applying Lemma 3 to $\mathcal{C}_{0}=\mathcal{C}_{\mathbf{Q}_{0}}, \mathcal{C}_{0}^{\prime}=\mathcal{C}_{\mathbf{P}_{1}}$, and $\overline{\mathcal{C}}=\mathcal{L}_{0}$ we find the following.
Lemma 4: For any differentiable curve $\mathcal{L}_{0}$ passing transversely through $\mathcal{U}$ at a point $\mathbf{z}_{\mathcal{U}}$, the capture zones $C_{n}$ intersect $\mathcal{L}_{0}$ for all $n$ large enough. We choose $\epsilon_{n}$ to be the connected component of $C_{n} \cap \mathcal{L}_{0}$ closest to $\mathbf{z}_{\mathcal{U}}$ (as measured along $\mathcal{L}_{0}$ ). The $\epsilon_{n}$ converge upon $\mathbf{z}_{\mathcal{U}}$ with the Euclidean length $\lambda_{n}$ of $\epsilon_{n}$ satisfying

$$
\lim _{n \rightarrow \infty} \lambda_{n} \alpha^{n}=A\left(\mathbf{z}_{\mathcal{U}}, \hat{\mathbf{t}}\right) s\left(C_{1}\right) \neq 0,
$$

where $s\left(C_{1}\right)$ is the canonical length of the $\mathcal{S}$ boundary of $C_{1}$ [Eq. (5d)].

So long as $\rho\left(\mathbf{z}_{\mathcal{U}}\right) \neq 0$, Lemmas 2-4 hold when the Euclidean length $\lambda$ is replaced by the measure $\mu$ defined by Eq. (6), except that $A\left(\mathbf{z}_{\mathcal{U}}, \hat{\mathbf{t}}\right)$ is multiplied by $\rho\left(\mathbf{z}_{\mathcal{U}}\right)$. In particular, Eq. (B19) yields Eq. (8a)

$$
\lim _{n \rightarrow \infty} \mu_{n} \alpha^{n}=\left[A\left(\mathbf{z}_{\mathcal{U}}, \hat{\mathbf{t}}\right) \rho\left(\mathbf{z}_{\mathcal{U}}\right)\right] s\left(C_{1}\right) \equiv K_{\mu} \neq 0,
$$

where $\mu_{n}$ is the measure of $\epsilon_{n}$.

Between $\epsilon_{n}$ and $\epsilon_{n+1}$ lies a gap on $\mathcal{L}_{0}$ with measure $\gamma_{n}$. Applying Lemma 3 to $\mathcal{C}_{0}=\mathcal{C}_{\mathbf{P}_{1}}$ and $\mathcal{C}_{0}^{\prime}=\mathcal{M}\left(\mathcal{C}_{\mathbf{Q}_{0}}\right)$ yields Eq. (8b),

$$
\lim _{n \rightarrow \infty} \gamma_{n} \alpha^{n}=\left[A\left(\mathbf{z}_{\mathcal{U}}, \hat{\mathbf{t}}\right) \rho\left(\mathbf{z}_{\mathcal{U}}\right)\right] s\left(E_{1}\right) \equiv K_{\gamma} \neq 0,
$$

from which also follows Eq. (9a),

$$
\lim _{n \rightarrow \infty} \frac{\mu_{n}}{\gamma_{n}}=\frac{s\left(C_{1}\right)}{s\left(E_{1}\right)}=\frac{s\left(C_{0}\right)}{s\left(E_{0}\right)}=\frac{K_{\mu}}{K_{\gamma}} \equiv \chi \neq 0 .
$$

Similarly, Lemma 2 applied to $\mathcal{C}_{0}=\mathcal{C}_{\mathbf{P}_{1}}$ implies Eq. (8c),

$$
\lim _{n \rightarrow \infty} \delta_{n} \alpha^{n}=\left[A\left(\mathbf{z}_{\mathcal{U}}, \hat{\mathbf{t}}\right) \rho\left(\mathbf{z}_{\mathcal{U}}\right)\right] s\left(\mathbf{P}_{1}\right) \equiv K_{\delta} \neq 0,
$$

where $\delta_{n}$ is the measure between $\epsilon_{n}$ and $\mathbf{z}_{\mathcal{U}}$. From Eq. (B23) follows Eq. (9b),

$$
\lim _{n \rightarrow \infty} \frac{\mu_{n}}{\delta_{n}}=\frac{s\left(C_{1}\right)}{s\left(\mathbf{P}_{1}\right)}=\frac{s\left(C_{0}\right)}{s\left(\mathbf{P}_{0}\right)}=\frac{K_{\mu}}{K_{\delta}} \equiv \phi \neq 0 .
$$

The Epistrophe Theorem requires studying intersections of the pre-iterates of $E_{0}$ with $\mathcal{L}_{0}$. In the preceding results, we have studied intersections of the forward iterates of $C_{0}$ with $\mathcal{L}_{0}$. One may, of course, translate between these two viewpoints by replacing $\mathcal{M}$ with $\mathcal{M}^{-1}$.

The final equality for $\phi$ follows from

$$
u\left(\mathbf{P}_{0}\right)=\sum_{n=0}^{\infty}\left[u\left(C_{0}\right)+u\left(E_{0}\right) \alpha^{-1}\right] \alpha^{-n}=u\left(C_{0}\right) \frac{\alpha+\chi}{\alpha-1} .
$$

${ }^{1}$ C. Jaffé, S. D. Ross, M. W. Lo, J. Marsden, D. Farrelly, and T. Uzer, Phys. Rev. Lett. 89, 011101 (2002).

${ }^{2}$ C. M. Marcus, A. J. Rimberg, R. M. Westervelt, P. F. Hopkins, and A. C. Gossard, Phys. Rev. Lett. 69, 506 (1992); C. M. Marcus, R. M. Westervelt, P. F. Hopkins, and A. C. Gossard, Chaos 3, 643 (1993); H. Ishio and J. Burgdörfer, Phys. Rev. B 51, 2013 (1995); X. Yang, H. Ishio, and J. Burgdörfer, ibid. 52, 8219 (1995); C. D. Schwieters, J. A. Alford, and J. B. Delos, ibid. 54, 10652 (1996).

${ }^{3}$ J. U. Noeckel and A. D. Stone, Nature (London) 385, 45 (1997).

${ }^{4}$ M. J. Davis and S. K. Gray, J. Chem. Phys. 84, 5389 (1986); M. J. Davis, ibid. 83, 1016 (1985). 
${ }^{5}$ A. Tiyapan and C. Jaffé, J. Chem. Phys. 99, 2765 (1993).

${ }^{6}$ A. Tiyapan and C. Jaffé, J. Chem. Phys. 101, 10393 (1994).

${ }^{7}$ A. Tiyapan and C. Jaffé, J. Chem. Phys. 103, 5499 (1995).

${ }^{8}$ P. M. Koch and D. R. Mariani, Phys. Rev. Lett. 46, 1275 (1981); see also A. Mühlpfordt, U. Even, E. Rabani, and R. D. Levine, Phys. Rev. A 51, 3922 (1995)

${ }^{9}$ C. Jaffé, D. Farrelly, and T. Uzer, Phys. Rev. A 60, 3833 (1999).

${ }^{10}$ C. Jaffé, D. Farrelly, and T. Uzer, Phys. Rev. Lett. 84, 610 (2000).

${ }^{11}$ C. Jaffé and T. Uzer, J. Phys. Chem. A 105, 2783 (2001).

${ }^{12} \mathrm{~S}$. Wiggins, L. Wiesenfeld, C. Jaffé, and T. Uzer, Phys. Rev. Lett. 86, 5478 (2001).

${ }^{13}$ G. M. Lankhuijzen and L. D. Noordam, Phys. Rev. Lett. 76, 1784 (1996).

${ }^{14}$ F. Robicheaux and J. Shaw, Phys. Rev. Lett. 77, 4154 (1996); Phys. Rev. A 56, 278 (1997).

${ }^{15}$ S. Wiggins, Chaotic Transport in Dynamical Systems (Springer, New York, 1992).

${ }^{16}$ E. A. Jackson, Perspectives of Nonlinear Dynamics, Vol. II (Cambridge University Press, Cambridge, 1990).

${ }^{17}$ M. Courtney, H. Jiao, N. Spellmeyer, D. Kleppner, J. Gao, and J. B. Delos, Phys. Rev. Lett. 74, 1538 (1995).

${ }^{18}$ G. Raithel and H. Walther, Phys. Rev. A 49, 1646 (1994); G. Raithel, M. Fauth, and H. Walther, ibid. 44, 1898 (1991).

${ }^{19}$ A. Holle, J. Main, G. Wiebusch, H. Rottke, and K. H. Welge, Phys. Rev. Lett. 61, 161 (1988).

${ }^{20}$ A. Kips, W. Vassen, W. Hogervorst, and P. A. Dando, Phys. Rev. A 58, 3043 (1998).

${ }^{21}$ U. Eichmann, K. Richter, D. Wintgen, and W. Sandner, Phys. Rev. Lett. 61, 2438 (1988).

${ }^{22}$ N. Spellmeyer, D. Kleppner, M. R. Haggerty, V. Kondratovich, J. B. Delos, and J. Gao, Phys. Rev. Lett. 79, 1650 (1997).

${ }^{23}$ M. L. Du and J. B. Delos, Phys. Rev. A 38, 1896 (1988).

${ }^{24}$ J. Gao and J. B. Delos, Phys. Rev. A 46, 1455 (1992).

${ }^{25}$ J. Main, G. Wiebusch, K. Welge, J. Shaw, and J. B. Delos, Phys. Rev. A 49, 847 (1994).

${ }^{26}$ We also have in mind applications to the semi-classical propagation of quantum wave functions, for which it is natural to consider an initial curve (Lagrangian manifold) in phase space (Ref. 56). Lines of initial conditions are also common in studies of classical chaotic scattering (as in Refs. 34-39).

${ }^{27}$ R. S. MacKay, J. D. Meiss, and I. C. Percival, Physica D 13, 55 (1984).

${ }^{28}$ V. Rom-Kedar, Physica D 43, 229 (1990).

${ }^{29}$ V. Rom-Kedar, Nonlinearity 7, 441 (1994).

${ }^{30}$ D. W. Noid, S. K. Gray, and S. A. Rice, J. Chem. Phys. 84, 2649 (1986).

${ }^{31}$ J.-M. Petit and M. Henon, Icarus 66, 536 (1986).

${ }^{32}$ B. Eckhardt and C. Jung, J. Phys. A 19, L829 (1986).
${ }^{33}$ B. Eckhardt, J. Phys. A 20, 5971 (1987).

${ }^{34}$ C. Jung and H. J. Scholz, J. Phys. A 20, 3607 (1987).

${ }^{35}$ B. Rückerl and C. Jung, J. Phys. A 27, 55 (1994).

${ }^{36}$ B. Rückerl and C. Jung, J. Phys. A 27, 6741 (1994).

${ }^{37}$ C. Lipp and C. Jung, J. Phys. A 28, 6887 (1995).

${ }^{38}$ C. Jung, C. Lipp, and T. H. Seligman, Ann. Phys. (Leipzig) 275, 151 (1999)

${ }^{39}$ T. Bütikofer, C. Jung, and T. H. Seligman, Phys. Lett. A 265, 76 (2000).

${ }^{40}$ P. Gaspard and S. Rice, J. Chem. Phys. 90, 2225 (1989).

${ }^{41}$ P. Gaspard, Chaos, Scattering and Statistical Mechanics (Cambridge University Press, Cambridge, 1998).

${ }^{42}$ J. Palis, Topology 8, 385 (1969).

${ }^{43}$ We thank R. MacKay for bringing this to our attention.

${ }^{44}$ R. W. Easton, Trans. Am. Math. Soc. 294, 719 (1986).

${ }^{45}$ An end point could also lie on $\mathcal{U}$, in which case it would be on the boundary of the complex and no epistrophe would converge upon it. See Fig. 1.

${ }^{46}$ Note that $P$ and $Q$ are canonical coordinates, whereas $\mathbf{P}_{n}$ and $\mathbf{Q}_{n}$ are points in the phase plane.

${ }^{47}$ Merriam-Webster's Collegiate Dictionary (Merriam-Webster, Springfield, MA, 1999).

${ }^{48}$ American College Dictionary (Random House, New York, 1947).

${ }^{49}$ In exceptional circumstances, there may be other intervals that survive. For example, it could happen that $\mathcal{L}_{0}$ coincides with the stable manifold over an interval of nonzero length, a nongeneric situation.

${ }^{50}$ Both $\mathrm{S}_{T}$ and $\mathbb{E}$ are subsets of both the line of initial conditions and the phase plane, but for topological purposes, we view them as subsets of $\mathcal{L}_{0}$. That is, an open interval of $\mathcal{L}_{0}$ is viewed as an open set, even though it is not an open subset of the phase plane.

${ }^{51}$ J. G. Hocking and G. S. Young, Topology (Addison-Wesley, Reading, MA, 1961), pp. 97-100.

${ }^{52}$ K. Falconer, Fractal Geometry: Mathematical Foundations and Applications (Wiley, Chichester, 1990); H.-O. Peitgen, H. Jürgens, and D. Saupe, Chaos and Fractals: New Frontiers of Science (Springer, New York, 1992).

${ }^{53}$ K. R. Meyer, Trans. Am. Math. Soc. 149, 95 (1970).

${ }^{54}$ K. R. Meyer and G. R. Hall, Introduction to Hamiltonian Dynamical Systems and the N-Body Problem (Springer, New York, 1992), pp. 224225.

${ }^{55}$ J. Moser, Commun. Pure Appl. Math. 9, 673 (1956).

${ }^{56}$ V. P. Maslov and M. V. Fedoriuk, Semi-classical Approximation in Quantum Mechanics (Reidel, Boston, 1981); J. B. Delos, Adv. Chem. Phys. 65, 161 (1966); M. V. Berry, Proc. R. Soc. London, Ser. A 413, 183 (1987). 